auch ein wenig an Wunder zu glauben. Es erweist sich damit als eines der ehrlichsten Bücher über die Gegenstände, die das Interesse dieser Zeitschrift finden. Der Rezensent meint feststellen zu dürfen, daß er ein sehr gutes Buch gelesen hat.

Dieter Schröder

\section{JeAn Vergeot}

Les Plans dans le Monde

(Preface d' Emile Roche, Postface de Rene Montjoie) Editions France-Empire, Paris 1970, $578 \mathrm{~S}$.

Ein Buch, das es auf 578 Seiten unternimmt, die Wirtschaftsplanung auf der ganzen Welt vergleichend darzustellen, darf eines aufmerksamen Interesses sicher sein, wird aber auch von vornherein manchen Bedenken begegnen. Diese ergeben sich einmal aus der Fülle des Stoffes. Der Verfasser behandelt zwar nicht alle Staaten der Erde, aber doch eine sehr weitgespannte Auswahl: die EWG-Mitglieder, von den übrigen kapitalistischen Staaten Großbritannien, die USA, Norwegen, Schweden, Spanien und Japan, als Beispiele für die verschiedenen kommunistischen Systeme die UdSSR, China und Jugoslawien und unter den Entwicklungsländern Indien und - jeweils ein wenig global - die frankophonen afrikanischen Staaten und den lateinamerikanischen Kontinent. Ein solches Programm läßt sich natürlich in diesem Rahmen nur bei äußerster Beschränkung auf das Notwendigste durchführen. Wenn man bereit ist, diese Voraussetzung anzuerkennen, und an das Buch keine falschen Ansprüche $\mathrm{zu}$ stellen, so findet man in den Länderberichten jeweils einen sehr wertvollen Überblick über die verschiedenen wirtschaftspolitischen Ausgangslagen, Organisation und Verfahren der Wirtschaftsplanung und deren erkennbare Ergebnisse.

Bezweifelt könnte jedoch auch werden, ob es überhaupt sinnvoll ist, die Wirt- schaftsplanung im Osten, Westen und "Süden" zusammen zu behandeln. Während es keiner Rechtfertigung für eine Zusammenstellung der westlichen Versuche bedarf, Marktwirtschaft und Planung miteinander $\mathrm{zu}$ verbinden ${ }^{1}$, sind wirtschaftliche und verfassungsrechtliche Situation der im angezeigten Buch behandelten Länder so unterschiedlich, daß die Fruchtbarkeit einer vergleichenden Darstellung fragwürdig ist. Immerhin ist in der Grundidee der Planung - die Wirtschaftsentwicklung nicht sich selbst $\mathrm{zu}$ überlassen - ein Minimum an Gemeinsamkeit gewährleistet. Dasselbe gilt, wie der Verfasser mit Recht feststellt (S. 506 f.), für die mit der Planung verfolgten Ziele (Vollbeschäftigung, Wachstum, außenwirtschaftliches Gleichgewicht und Preisstabilität sowie gerechte Einkommenverteilung und ausgeglichene Entwicklung der verschiedenen Landesteile). Gerade die Identität von Zielen rechtfertigt aber im Grunde immer einen Vergleich der verschiedenen Wege. Außerdem stellt der Verfasser eine gewisse Angleichung der Systeme fest (S. 499): die Planungsfeindlichkeit in Staaten wie der Bundesrepublik Deutschland und den USA läßt nach und gleichzeitig werden die Planungssysteme des Osten liberalisiert (zusammenfassend S. 504). Diese Entwicklung (aus der der Verfasser ein wenig vereinfachend die Richtigkeit des „mittleren" französischen Weges folgert, S. 545) erleichtert einen Vergleich natürlich. Auch die dritte Gruppe, die Entwicklungsländer, fällt nicht aus dem Rahmen, da diese weitgehend Planungsverfahren der Industriestaaten rezipiert haben. Eine wichtige Frage wäre in diesem Zusammenhang allerdings, wie weit die rezipierten Systeme für die Bedürfnisse dieser Länder adäquat sind. $\mathrm{Da}$ die frankophonen afrikanischen Staaten ihre Wirtschaftsplanung weitgehend der französischen "planification" nachbilden, kann man natürlich als Beweis der Güte des französischen Systems nehmen, näher liegt es jedoch, zu bezweifeln, daß

1 Vgl. Karlheinz Kleps, Langfristige Wirtschaftspolitik in Westeuropa, Freiburg 1966. 
diese Staaten damit schon die ihnen gemäßen Verfahren für die Planung ihrer wirtschaftlichen Entwicklung gefunden haben. Das ist jedoch nötig, wenn Wirtschaftsplanung für diese Länder, wie der Verfasser richtig feststellt (S. 505) unerläßlich ist (und daher vielfach zur Voraussetzung für die Gewährung von Entwicklungshilfe gemacht wird).

Ein Vergleich von Planungssystemen über die ganze Welt ist nach allem sinnvoll. $\mathrm{Da}$ er schwer ist, macht das Buch von Vergeot selbst deutlich. Insbesondere im zweiten, vergleichenden Teil (S. 498 ff.) liegt das Schwergewicht nämlich eindeutig bei den westlichen Planungssystemen. Dieser Teil ist besonders wichtig, da der Verfasser hier über die bei „vergleichenden“ Darstellungen of $t$ übliche bloße Addition von Länderberichten (zwischen denen eine Beziehung allenfalls durch ein kurzes Voroder Nachwort hergestellt wird) hinausgeht und konkret die verschiedenen Planungsmethoden nach ihrer Organisation, Durchführung und Kontrolle vergleicht. Eine derartige Darstellung erfordert eine gewisse Vergleichbarkeit nicht nur in Grundgedanken und Ziel der Wirtschaftsplanung, sondern auch in ihrem Aufbau. Daß Vergeot sich hier weitgehend auf einen Vergleich der westlichen Länder beschränkt und die östlichen nur zur Abrundung heranzieht, ist daher verständlich, findet seine Begründung darüber hinaus aber auch darin, daß das Buch in eine Auswertung der ausländischen Erfahrungen für die französische Planung einmündet. Es wäre zu wünschen, daß Nutzen aus diesem Buch, in das der Autor den Sachverstand einer langjährigen maßgeblichen Mitwirkung in der französischen "planification" eingebracht hat, nicht nur in Frankreich gezogen wird.

Brun-Otto Bryde
The Cambridge History of Islam

P. M. Holt, A. K. S. Lambton, B. Lewis (eds.), Cambridge University Press, 1970, Bd. 1: The Central Islamic Lands, 815 S., ₹ 6.

Bd. 2: The further Islamic Lands, Islamic Society and Civilization, 966 S. £ 7

Drei Historiker der Universität London haben es mit dem vorliegenden Werk über die komplexe Geschichte des Islams unternommen, diesen als ein kulturelles Ganzes zu erfassen und dabei alle Aspekte einzubeziehen: politische, theologische, philosophische, wirtschaftliche, wissenschaftliche, militärische und künstlerische. Man war dabei bemüht, nicht nur eine Aneinanderreihung von Fakten zu geben, also nicht nur ein Nachschlagewerk zu verfassen, sondern ein Buch zu schaffen, welches der kulturgeschichtlich Interessierte kontinuierlich lesen kann. Eine so weit gesteckte Aufgabe setzt ein Team-work voraus, weshalb die einzelnen Kapitel (je 23 in Bd. 1 und Bd. 2, sowie 2 Exkurse) von den verschiedensten Spezialisten aus aller Welt bearbeitet wurden, deren Beiträge zu synchronisieren, allein schon eine mühevolle Leistung darstellt.

Das Werk umfaßt je Band 4 Teile. Im ersten Band, der die islamischen Kerngebiete umfaßt, wird zuerst das Heraufkommen und die Herrschaft der Araber behandelt (S. 1-139), sodann das Eindringen zentralasiatischer Steppenvölker in den Nahen Osten (S. 143291), gipfelnd in der Periode der türkischen Ottomanen (S. 295-523) und endend mit der Entwicklung bis in die Gegenwart (S. 527-730). - Der zweite Band ist in Teil 5-7 Indien, Südostasien und Afrika außerhalb Ägyptens gewidmet (S. 1-439) und schließt ab mit dem 8. Teil über islamische $\mathrm{Ge}-$ sellschaft und Zivilisation (S. 443-889), der auch eine größere Zahl von Abbildungen aus islamischer Kunst enthält.

Die beiden Bände sind voneinander unabhängig und auch einzeln erwerbbar. Sie sind getrennt paginiert, haben getrennte Register und Bibliographien; 